\title{
Prostatic Adenocarcinoma: A Grading from Gleason to the New Grade-Group System: A Historical and Critical Review
}

\author{
Muhammad Abrar Barakzai*
}

\begin{abstract}
The introduction of the Gleason grading system revolutionised prognostic parameters and determination of patient treatment regiments for prostatic adenocarcinomas, and has become synonymous with prostate cancer, almost universally applied in clinical settings to predict radical prostatectomy specimen findings, potential biochemical failure, local recurrences, lymph nodes or distant metastases in patients not receiving any treatment as well as those receiving treatment including radiation therapy, surgical treatment such as radical prostatectomy and other therapies etc,. However, characterisation and classification of prostate cancer is very different compared to 40-50 years ago when Gleason scores were first introduced. Despite this radical shift in classification, the Gleason system has remained one of the most important prognostic factors in prostate cancer, only possible as a result of timely and appropriate modifications to this characterisation system made in 2005 and 2014. However, even after these modifications, certain limitations of the Gleason system remain, due to which a new prostate cancer prognostic grade group system was introduced in 2014, which was widely accepted in the 2014 ISUP consensus conference, and incorporated into the WHO classification of thetumor of the Urinary System and Male Genital Tract in 2016. Herein, this article will discuss how this new prognostic grade group system, which is regarded as simpler and more accurate than the Gleason system risk stratification groups, will be used in conjunction with the Gleason system to improve patient prognosis and treatment.
\end{abstract}

Keywords: Prostate- Adenocarcinoma- Gleason grade

Asian Pac J Cancer Prev, 20 (3), 661-666

\section{Introduction}

The Gleason grading system for prostatic adenocarcinoma is synonymous with prostate cancer. It is almost universally applied in clinical setting both as a prognostic parameter and to determine patient treatment. It is an essential prognostic factor in predicting findings in radical prostatectomy specimen, biochemical failure, local recurrences, lymph nodes or distant metastases in patients not receiving any treatment, as well as those receiving treatment including radiation therapy, surgical treatment such as radical prostatectomy and other therapies etc. (Montironi et al., 2011; Delahunt et al., 2016). Clinicians can imply various tools for pathological staging and/or for determining prognosis following radical prostatectomy or radiotherapy. All these incorporate the Gleason score (Montironi et al., 2010).

The Gleason grading system is based on a study conducted by the Veterans Affairs Cooperative Urological Research Group (VACURG) between 1959 and 1964 which enrolled 270 men with prostate cancer. The purpose of this actually was to define optimal treatment for prostate cancer and the development of the grading system was one component of the above study. Dr Donald
F. Gleason was the trail Pathologist for the study. He was the Chief of Pathology and Laboratory Services at Veteran Administration Medical Centre in Minneapolis, Minnesota and a faculty member of the Department of Laboratory Medicine and Pathology at the University of Minnesota. The Gleason grading system is a histopathological grading system based on the architectural patterns of prostate cancer (Delahunt et al., 2012; Bailar et al., 1966; Barakzai et al., 2011)

The original Gleason Grading System and earlier modifications

Gleason decided that an ideal grading system should be based on low power assessment rather than detailed evaluation of nuclear features. He noted that "grading is performed under modification" (Gleason, 1966; Gleason, 1977). Thus, the Gleason grading of prostatic adenocarcinoma is typically performed using the $4 \mathrm{x}$ objective, but occasionally the higher modification $10 \mathrm{x}$ is required (Gordetsky et al., 2016).

Gleason initially observed that there were nine histological pattern of prostatic adenocarcinoma, (Delahunt et al., 2016) later however, he determined that some patterns could be combined. He found that

Department of Pathology, College of Medicine, Alfaisal University, Riyadh, Saudi Arabia. *For Correspondence: abarakzai@alfaisal.edu 
most of thetumor had more than one histological patterns (two rather than one pattern) (Gleason and Mellinger, 1974). He thus created a five point (tier) grading scale incorporating both the predominant and the less dominant histological pattern (major and minor, primary and secondary, and ultimately a score based on adding the two patterns.

The score is arranged from 2 to 10 (Gleason, 1992). This original Gleason grading system, which was solely based on the architectural pattern of thetumor, was thus created in 1966 (Gleason, 1966).

The patterns 1 to 3 representedtumors which most closely resembled normal prostate gland while patterns 4 to 5 representedtumors with increasingly abnormal glandular architecture. An innovative aspect of the system was that rather than assigning the worst grade as the grade of the carcinoma, the grade was defined as "the sum of the two most common patterns", and reported as the Gleason score (Barakzai et al., Gleason, 1966). Gleason's original study demonstrated a progressive increase in cancer specific mortality with an increase in the Gleason score. For ease of grading, Dr Gleason demonstrated the 5 architectural patterns in a single diagram (Gleason and Mellinger, 1974).

By 1974, Gleason and VACURG expanded their study to 1,032 patients. Gleason pattern 4 was described in a figure legend "as raggedly infiltrating fused glandulartumor, frequently with pale cells may resemble hypernephroma of kidney" The Gleason system was revalidated and further refined, through analysis of 2,911 patients, Mellinger who described the papillary and cribriformtumor under Gleason pattern 3 as having "a smooth and usually rounded edge." Mellinger recognized pattern 3 (Figure 1) as the commonest pattern (Gleason and Mellinger, 1974; Gleason, 1992; Mellinger, 1977; Mellinger et al., 1967). In 1977, Gleason also provided additional comments. He noted that "an occasional small area of fused glands did not change a pattern 3tumor to pattern 4 (Figure 2). A small focus of disorganised cells did not change a pattern 3 or 4 to pattern 5 (Figure 3)". He further noted that occasionally small areas of a third pattern were observed. These were the first comments relating to a tertiary pattern in some prostatic adenocarcinoma (Gleason, 1977).

In 1992, the patterns 3, 4, and 5 were subdivided on morphological grounds. This was the final version of the Gleason grading system modified by Gleason himself. In the 1992 version, poorly formed glands were classified as pattern $3 \mathrm{~B}$ while glands showing a cribriform pattern were included in both 3C and 4A. In the 1992 the modification of the Gleason grading system, prostatic adenocarcinoma was divided into Grades 1, 2, 3A, 3B, 3C, 4A, 4B, 5A and 5B. Papillary and cribriform glands were included in grades $3 \mathrm{C}$ (when arranged in smooth, sharply circumscribed rounded cylinders and masses) and 5A (when also arranged in smooth, rounded masses but more solid than 3C with central necrosis) (Gleason, 1992).

\section{The 2005 ISUP modified Gleason Grading system}

Prostate carcinoma had become a very different disease by 2005 compared to the previous years (since the inception of the Gleason grading system). In 1960s, there was no screening for prostatic carcinoma except by digital rectal examination (DRE). Serum PSA (Prostate specific antigen) had not yet been discovered. In Gleason's 1974 study, the vast majority of the patients had advanced cancer with either extra-prostatic extension on DRE or distant metastases. Only $6 \%$ of the patients had non palpabletumor diagnosed by TUR (transurethral resection) while $8 \%$ were diagnosed with a localized nodule on DRE (Rodolfo et al., 2011; Hodge et al., 2007).

The method of obtaining prostate biopsies was also very different. Typically, only couple of thick gauge needle biopsies were directed into an area of palpable abnormality, usually through the perineum. The use of 18 gauge thin needles and the concept of sextant needle biopsies to sample the prostate more extensively were only developed in the 1980s. Therefore, the grading of prostate cancer in thin cores and in multiple cores from different areas of the prostate were not a major issue or concern in Gleason's era (Rodolfo et al., 2011).

Radical prostatectomies (RPs) were relatively uncommon. Prostates were not as often removed intact and were not processed entirely or as systematically as they are processed now. Further issues relating to radical prostatectomy specimens, such as, grading of multiple nodules within the same prostate or presence of tertiary pattern in radical prostatectomy (RP) specimens were not addressed (Rodolfo et al., 2011).

The use of serum PSA as a screening method for prostate cancer led to detection of the cancers at an earlier, less advanced stages. This led to greater emphasis on accurately identifying patients with early disease who were more likely to benefit from attempts at curative therapies or who could be offered delayed treatment (active surveillance) (Delahunt et al., 2012).

Immunohistochemistry was not used in the microscopic examination of thetumors. It is likely that with immune-staining for basal cells, many of the Gleason's original $1+1=2$ prostate cancers would today be diagnosed as adenosis (atypical adenomatous hyperplasia) that is a benign lesions (Berney, 2007).

In view of all these factors, a need was felt to review the prognostic significance of the Gleason grading system and to revise the grading criteria. It must, however, be emphasized before discussing the revisions that this Gleason grading system has remained, and until now, one of the most powerful prognostic factors in prostatic carcinoma. It has remained so by making gradual and timely adaptations to accommodate the changing practices in medicine. Certain aspects of the original Gleason system are interpreted differently in today's practice (Amin et al., 2005).

Thus, the International Society of Urological Pathology (ISUP) convened a conference in San Antonio, Taxes, USA in 2005 to achieve consensus on the controversial areas within the Gleason system.

The conference was preceded by an international consensus meeting on "International consultation on predicators of patient outcome in prostate cancer". In Stockholm, Sweden which was sponsored by the World Health Organization (WHO) (Amin et al., 2005). 
National groups, independent from ISUP activities, had already undertaken the work of revising the Gleason system and submitted proposals that preceded those in the 2005 modified ISUP Gleason grading system (Epstein et al., 2005; Zabera et al., 2009; Griffiths et al., 2006; Melia et al., 2006).

The modifications in the 2005 system are summarised as follow:

- A Gleason score of $1+1=2$ should not be diagnosed regardless of the type of specimen with extremely rare exception.

- The diagnosis of Gleason scores 2 to 5 should no longer be made on needle biopsies. Thus a scale from 2 to 10 is no longer applicable. This is because of poor reproducibility and poor correlation with the radical prostatectomy grades.

- A diagnosis of Gleason scores $2-5$ is misleading for both clinicians and patients as nearly all cases show higher grades at resection (grades 2 to 5 simply represent the deceptively bland edge of a higher grade lesions).

Since 2005, there has been a dramatic decrease in the diagnosis of Gleason score 2 to 5 as compared to a rate of over 22\% in prostatic biopsies in 1994.

- It was decided that "individual cells" will not be allowed in Gleason grade 3. Pattern 3 morphology will consist of well-formed acini only (Figure. 1).

- It was further decided that the vast majority of cribriform pattern will be diagnosed as Gleason Grade 4 and only rare cribriform cancers could satisfactorily fit into Gleason pattern $3 \mathrm{C}$.

- For needle biopsies, it was decided that secondary pattern, if of a higher grade, would always be included in the Gleason score even if its quality were very low as long as it can be identified at low to medium magnification. In essence, secondary patterns of a higher grade on needle biopsy should be reported as a part of Gleason score whatever their quantity. On the other hand, if the secondary pattern in the needle biopsy is of lower grade compared to primary (major) pattern, it need not be reported and should be ignored if it occupies less than $5 \%$ of thetumor.

- In the event of presence of tertiary grade, it was decided that for needle biopsies tertiary grade should be part of the Gleason score if greater than the secondary

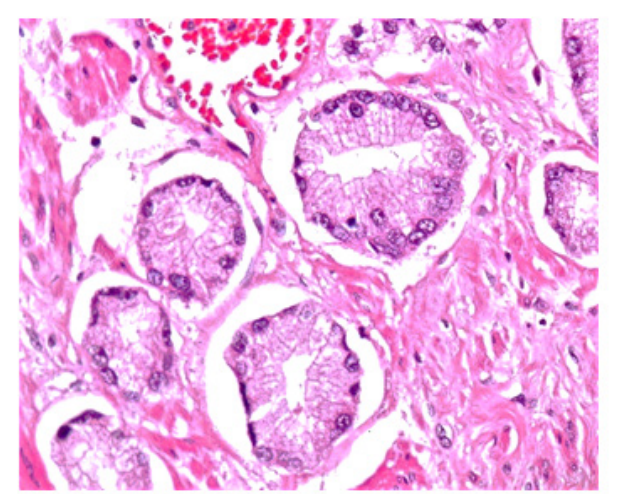

Figure 1. High Magnification Image Showing Well Former Glandular Lumina, a Pattern Consistent with Gleason Score 3+3=6(Grade group 1) Adenocarcinoma of the Prostate (H\&E x 400).
Table 1. Prostate Adenocarcinoma Grade Group Versus Gleason Score

\begin{tabular}{l}
\hline S.No Grade Group Gleason Score \\
\hline 1 Grade Group 1 Gleason score $\leq 6$ \\
2 Grade Group 2 Gleason score $3+4=7$ \\
3 Grade Group 3 Gleason score $4+3=7$ \\
4 Grade Group 4 Gleason score $4+4=8,3+5=8,5+3=8$ \\
5 Grade Group 5 Gleason score $9-10$ \\
\hline
\end{tabular}

pattern. Thus, in presence of tertiary pattern on needle biopsies, it was decided that the primary (major) pattern plus the highest grade will be part of the Gleason score. However, in the presence of the tertiary pattern in radical prostatectomy specimens, it was decided that the Gleason score will be based on the primary (major) and secondary (minor) pattern while the presence of the tertiary pattern will be included in the report as a comment if it is less than $5 \%$ of the wholetumor, and will be regarded as the secondary pattern.

The 2005 modified Gleason grading system also decided that each core in needle biopsies should be consigned individual Gleason score and recommended that cores taken from different areas of the prostate should be sent in separate containers (specific site/location in the prostate clearly labelled or different colour tubes for different areas of the prostate) to achieves this goal. It also included an option for the pathologist to give an overall score if the pathologist so desires.

The 2005 modification also recommended that in presence of fragmented cores, when the pathologist is not sure whether he/she is looking at a single core or separate cores, only an overall score should be assigned for all the pieces in that particular container. Studies has demonstrated that when different cores has different grades, the highest Gleason score correlates best with the Gleason score on radical prostatectomy specimens as compared to an average or overall score.

The 2005 modification also emphasized the importance of the percentage of Gleason 4 pattern (Figure 2) in Gleason score 7 tumors, since studies showed that carcinomas with Gleason score $4+3=7$ behaved significantly more aggressively than carcinomas with Gleason score $3+4=7$. In $4+3=7$ tumors, more than $50 \%$ of the cancers has a Gleason 4 pattern while in $3+4=7$, more than $50 \%$ of the cancers have Gleason 3 pattern. Since the pattern 4 confers a worse prognosis (higher the proportion of pattern 4 , worse the prognosis), its relative amount was believed to be important in Gleason 7 carcinomas. The 2005 grading system however did not decide whether the actual percentage of pattern 4 in Gleason score 7 tumors should be included in the report or not and gave the pathologist the option to include the percentage in the report if they so desired. At that time, studies on this issue were going on and it was yet to be conclusively proved as being statistically significant (although widely believed). A consensus was also reached in the 2005 Gleason grading system that ill-defined glands with poorly formed lumina will be included in Gleason pattern 4 (Epstein et al., 2005).

The 2005 modifications of the Gleason grading

Asian Pacific Journal of Cancer Prevention, Vol 20 


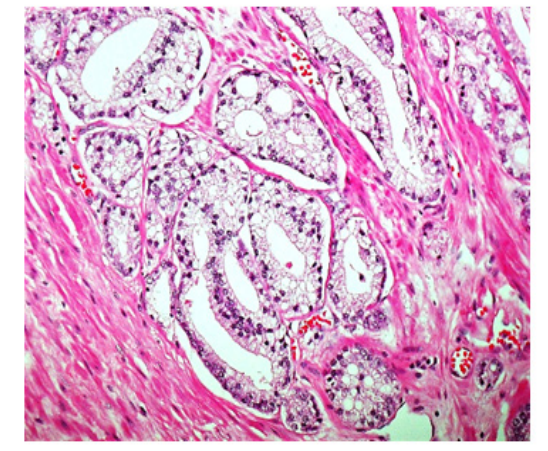

Figure 2. High Magnification Image Showing a Few Glandular Lumina with Focal Area of Loss of Glandular Differentiation, a Pattern Consistent with Gleason Score $4+3=7$ (Grade group 3) Adenocarcinoma of the Prostate ( $\mathrm{H}$ and $\mathrm{E} \mathrm{x} 400)$.

system resulted in a decrease in the diagnosis of pattern 3 (since its definition was limited) and an increase in the diagnosis of pattern 4 (as its definition was expanded). Thus the percentage of cases with Gleason score 6 decreased on needle biopsies while the percentage of cancers with Gleason score 7 increased correspondingly. Another important result of the 2005 modification of the Gleason grading system was increased agreement between the Gleason score on needle biopsies and the Gleason score on radical prostatectomy. Previously, there was considerable disagreement between the tumor grades on needle biopsies and radical prostatectomies, principally owing to the fact that the diagnosis of low grades (scores 2 to 5) was allowed. The disagreement mainly was in the under grading rather than over grading and the under grading mostly resulted due to difficulty in recognizing an infiltrative growth pattern or small areas of fused glands on needle biopsies. Yet another significant result of the 2005 modification was a significant improvement in the overall inter-observer reproducibility compared to the past. This improved inter-observer reproducibility was mainly due to two factors, one, changes and expansion in the definition of pattern 4; and two, greatly decreased diagnosis of low grade, Gleason sore 2 to 5 carcinomas. Of the two, various authors have placed greater emphasis on the changes and expansion of the pattern 4 carcinoma definition (Billis et al., 2008; Helpap and Egevad, 2006).

The 2005 the ISUP grading system recommended that in radical prostatectomy specimens, each dominant tumor nodule should be assigned a separate Gleason score. Mostly one or two dominant tumor nodules are found in radical prostatectomy specimens and assigning separate Gleason scores to each nodule is important when different nodules have different Gleason scores. While in most cases, the most dominant nodule usually has the highest grade, in some cases, a smaller nodule may have a higher score than the dominant nodule. In such cases, the patient's prognosis depends on the nodule with the highest score (Epstein et al., 2005).

Although, the 2005 modifications in the Gleason grading system improved its limitations and resulted in better correlation with patient outcomes, studies have also made it clear that several aspects of the Gleason system remained unresolved and needed to be refined further.

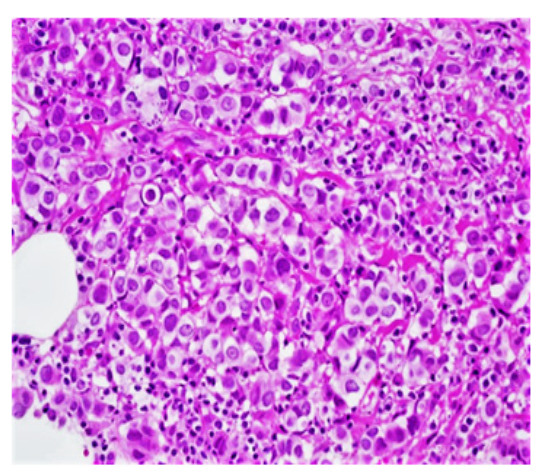

Figure 3. High Magnification Image Showing Solid Pattern of Growth and Lack of any Glandular Differentiation, a Pattern Consistent with Gleason Score $5+5=10$ (Grade group 5) Adenocarcinoma of the Prostate (H and E x 400).

Gleason system has become more complex and confusing for both the clinicians and patients themselves. Various modifications over the years have made the Gleason system markedly different from the original system in its use and application. Nowadays, the lowest score attained on needle biopsies is 6 whereas the scale ranges from 2 to 10. In addition, certain patterns of Gleason score 6 are now considered pattern 7. Thus, as discussed above, Gleason 6 carcinomas, as diagnosed currently, have a better prognosis than the Gleason 6 cancers diagnosed in the past. Based on the updated criteria, organ confined, margin negative (on radical prostatectomy), Gleason score 6 cancer is almost $100 \%$ curable. Studies have demonstrated that Gleason 6 cancers do not recur after radical prostatectomy and do not metastasize to lymph nodes. However, since score 6 is midway on the scale 2 to 10 , it gives a false and misleading impression to both clinicians and patients who may assume and interpret a score 6 cancer to be in the midrange prognostically. This is in marked contrast to the now proven fact that grade 6 cancers have the best prognosis.

Even after the 2005 modifications in the Gleason system, a score of 7 (whether $3+4=7$ or $4+3=7$ ) was taken as the same prognostically. The National Comprehensive Cancer Network (NCCN) and American Classification system which are the most common risk stratification systems for prostate cancer categorize prostate cancer as low risk (scores 2 to 6), intermediate risk (score 7) and high risk (scores 8 to 10). This system considers $3+4=7$ and $4+3=7$ equally as an intermediate risk tumor. However, multiple recent studies have shown that Gleason score $4+3=7$ cancers have a much worse prognosis compared to Gleason scores $3+4=7$ cancers. Similarly, in the same system, scores 8,9 and 10 are grouped together as high risk but recent studies have demonstrated that patients with Gleason score 8 cancers have significantly better prognosis when compared to Gleason score 9 and 10 cancers (Pierorazio et al., 2013; Burdick et al., 2009; Chan et al., 2000; Kang et al., 2007).

\section{The 2014 ISUP modified Gleason Grading system}

To effectively address these deficiencies, the International Society for Urologic Pathologists (ISUP) held a consensus conference in Chicago in 2014 in which 
67 urologic pathologists from 17 countries with expertise in prostate cancer pathology participated. In addition, the conference was attended by urologists, medical oncologists, and radiation oncologists, etc. Prior to this, a new grading system was proposed in 2013 by a group from Johns Hopkins Hospital. This new grading system was based on a study led by Jonathan Epstein, which utilized clinical data from the Johns Hopkins Hospital. This system was then validated by a multi institutional study including Johns Hopkins Hospital, Memorial Sloan-Kettering Cancer Centre, Cleveland Clinic, University of Pittsburgh and Karolinska Institute, which included more than 20,000 men with prostate cancer. This new contemporary prostate cancer grading system stratifies prostate cancer into prognostic grade groups which are based on prognostic behaviour of prostate cancers of various Gleason scores. These prognostic grade groups were separated on the basis of probability of 5-year biochemical recurrences (BCR) free progression following radical prostatectomy (RP). According to this grading system, prognostic grade group 1 (Figure 1) includes all prostate cancers with Gleason scores 6 (or less) which are indolent cancers in which only active surveillance can be considered. Prognostic grade group 1 score 6 cancers have individual discrete, well-formed glands and demonstrate a $96 \%$ probability of 5 year BCR free progression after RP. Prognostic grade group 2 comprises tumors with score $3+4=7$ which are predominantly composed of well-formed glands with lesser component of poorly formed / fused / cribriform glands. These prognostic grade groups 2 demonstrate an $88 \%$ probability of 5-year BCR free progression following RP. Prognostic grade group 3 (Figure. 2) comprises cancers with score $4+3=7$ which predominantly show poorly formed / fused / cribriform glands with a lesser component of well-formed glands. Prognostic grade group 3 cancers demonstrate a 63\% probability of 5 year BCR free progression after RP. Prognostic grade group 4 comprises cancers with score $4+4=8$ or $3+5=8$ or $5+3=8$. These cancers either show only poorly formed / fused/ cribriform glands $(4+4=8)$, or they show predominantly well-formed glands and a lesser component lacking any glands $(3+5=8)$, or they predominantly lack glands and show only a lesser component of well-formed glands $(5+3=8)$. These prognostic grade group 4 (score 8 ) cancers behave similarly whether they are $4+4=8$ or $3+5=8$ or $5+3=8$ and demonstrate a $48 \%$ probability of 5 -year BCR free progression following RP. Prognostic grade group 5 (Figure. 3) comprises cancers with score $4+5=9,5+4=9$ and $5+5=10$. These tumors show complete lack of gland formation (or with necrosis) with poorly formed/fused/ cribriform glands $(4+5=9$ or $5+4=9)$ or complete lack of gland formation (Figure. 3) $(5+5=10)$. All these prognostic grade group 5 cancers behave similarly whether $4+5=9$ or $5+4=9$ or $5+5=10$ and demonstrate a $26 \%$ probability of 5 year BCR free progression after RP (Epstein et al., 2016).

This new prostate cancer grading system was accepted at the 2014 ISUP Consensus Conference where $90 \%$ of the participants voted in its support. It has been incorporated in the 2016 WHO book on "Tumors of the urinary system and male genital organs". However, the consensus conference recommended that at least for the near future, both the Gleason grading system and the new prognostic grade group system should be incorporated in prostate cancer reports. This was done because Gleason grading system occupies such a pivotal place in prostate cancer and it will be some time before the new system can become widely accepted and replace the Gleason system (Humphrey et al., 2016). Thus, for now, both grading systems will be used in conjunction. It needs to be mentioned here that the new prognostic grade groups have been shown to be more accurate than the Gleason system risk stratification groups ( 6 or less $7,8,9,10)$ and whether the patients have been treated by RP, radiation therapy and/or hormonal therapy. Thus the new system is not only simpler, but also reflects the prognosis more accurately. With this new system patients with prognostic grade group 1 tumor are reassured that they have the lowest grade tumor with the best prognosis in which only active surveillance is required in most cases, whereas in the past, patients (and clinicians) were apprehensive that they have at least an intermediate grade tumor since it is in the middle of a scale ranging from 2 to 10 . The new system separates Gleason $3+4=7$ (prognostic grade group 2) from $4+3=7$ (prognostic grade group 3) tumors which were lumped together in the Gleason grading system but actually show significant differences in prognosis with the latter being much more aggressive. Similarly while in the Gleason grading system tumors with scores 8, 9, 10 were lumped together, the new system separates Gleason score $8(4+4,3+5,5+3)$ tumors, which have a significantly better prognosis from score $9(4+5,5+4)$ and 10 tumors, which have considerably worse prognosis (Pierorazio et al., 2013; Epstein et al., 2016) (Table 1).

In addition, the 2014 ISUP consensus conference made several other recommendations:

- Since multiple studies demonstrated the importance of including percent of pattern 4 in all Gleason 7 scores, the conference recommended that percent pattern 4 be included in all reports of score 7 prostate carcinoma. Recording percent pattern 4 will identify borderline Gleason 7 tumors (borderline between $3+4$ and $4+3$ ) in which factors other than the Gleason grade may be important in determining the radiation therapy regimens.

- Both the 2014 (and the 2005) ISUP consensus conference recommended separate grading for each district dominant tumor nodule in RP specimens. Smaller nodules need not be graded unless they show a higher grade than the dominant nodules which is very uncommon.

- It recommended that all cancers with cribriform, fused and poorly formed as well as glomeruloid glands be graded as pattern 4 .

- The 2014 consensus conference recommended that the term "hypernephroid" may not be used anymore while diagnosis of Gleason pattern 4 composed of clusters of poorly formed or fused glands without any cribriform glands be made at a higher magnification i.e. 10X.

- It recommended that in addition to small, separate glands, borderline glands be allowed in Gleason 3 .

- It recommended that unequivocal comedo-necrosis, even if present only focally, be graded as pattern 5 . Similarly, solid nest with rosette like spaces also be 
considered as pattern 5 .

- The 2014 consensus conference recommended that tumors with borderline morphology between patterns 3 and 4 and in cases when they show crushing artefacts, the lower grade be favoured (Epstein et al., 2016; Humphrey et al., 2016; Epstein et al., 2016; Matoso et al., 2016).

In conclusion, the Gleason system remains and until today a powerful prognostic indicator of prostate cancer and continues to play an important role in the treatment of prostate cancer. Its deficiencies (owing to changes in the current clinical practices) were corrected at the 2005 and 2014 ISUP consensus conferences. However, it still has certain limitations owing to which a new prostate cancer grading system has been introduced and accepted. This system is not only simpler but it also improves the Gleason system more accurately predicting the prognosis of prostate cancer.

\section{Financial Disclosure}

None.

\section{Conflict of Interest}

None.

\section{References}

Amin M, Boccon GL, Egerad L, et al (2005). Prognostic and predictive factors and reporting of prostate carcinoma in prostate needle biopsy specimens. Scand J Urol Nephrol Suppl, 216, 20-33.

Bailar JC, Mellinger GT, Gleason DF (1966). Survival rates of patients with prostatic cancer, tumor stage, and differentiation-preliminary report. Cancer Chemother Rep, 50, 129-36.

Barakzai MA, Mubarak M, Kazi JI (2011). Histopathological lesions in transrectal ultrasound guided biopsies of prostate in patients with raised serum prostate specific antigen: A preliminary report. Nephro-Urol, 3,186-90

Berney DM (2007). The case for modifying the Gleason grading system. BJU Int, 100, 725-6.

Billis A, Guimaraes MS, Freitas LL, et al (2008). The impact of the 2005 international society of urological pathology consensus conference on standard Gleason grading of prostatic carcinoma in needle biopsies. $J$ Urol, 180, 548-52.

Burdick MJ, Reddy CA, Ulchaker J (2009). Comparison of biochemical relapse-free survival between primary Gleason score 3 and primary Gleason score 4 for biopsy Gleason score 7 prostate cancer. Int J Radiat Oncol Biol Phys, 73, 1439-45.

Chan TY, Partin AW, Walsh PC, Epstein JI (2000). Prognostic significance of Gleason score $3+4$ versus Gleason score $4+3$ tumor at radical prostatectomy. Urology, 56, 823-7.

Delahunt B, Miller RJ, Srigley JR, Evans AJ, Samaratunga H (2012). Gleason grading: past, present and future. Histopathology, 60, 75-86.

Delahunt B, Egevad L, Samaratunga H, et al (2016). Gleason and Fuhrman no longer make the grade. Histopathology, 68, 475-81.

Epstein JI, Allsbrook WC, Amin MB, Egevad LL (2005). The 2005 International Society of Urological Pathology (ISUP) Consensus Conference on Gleason Grading of Prostatic Carcinoma. Am J Surg Pathol, 29, 1228-42.

Epstein JI, Zelefsky MJ, Sjoberg DD, et al (2016). A contemporary prostate cancer grading system: A validated alternative to the Gleason score. Eur Urol, 69, 428-35.
Epstein JI, Egevad L, Amin MB (2016). The 2014 International Society of Urological Pathology (ISUP) Consensus Conference on Gleason Grading of Prostatic Carcinoma: Definition of Grading Patterns and Proposal for a New Grading System. Am J Surg Pathol, 40, 244-52.

Gleason DF (1966). Classification of prostatic carcinomas. Cancer Chemother Rep, 50, 125-8.

Gleason DF, Mellinger GT (1974). Prediction of prognosis for prostatic adenocarcinoma by combined histological grading and clinical staging. $J$ Urol, 111, 58-64.

Gleason DF (1977). Morphological grading and clinical staging of prostate carcinoma. In Tannenbaum Med. Urologic Pathology. The prostate. Philedelphia, lea and Feibger, pp 171-98.

Gleason DF (1992). Histologic grading of prostate cancer: a perspective. Hum Pathol, 23, 273-9

Gordetsky J, Epstein J (2016). Grading of prostatic adenocarcinoma: current state and prognostic implications. Diagn Pathol, 11, 1-8.

Griffiths DF, Melia J, McWilliam LJ, et al (2006). A study of Gleason score interpretation in different groups of UK pathologists: techniques for improving reproducibility. Histopathology, 48, 655-62.

Helpap B, Egevad L (2006). The significance of modified Gleason grading of prostatic carcinoma in biopsy and radical prostatectomy specimens. Virchows Arch, 449, 622-7.

Hodge KK, McNeal E, Terris MK, Stamey TA (1989). Random systematic versus directed ultrasound guided transrectal core biopsies of the prostate. $J$ Urol, 142, 71-4

Humphrey PA, Eguand L, Netfo GT, et al (2016). WHO Classification oftumors of the Urinary System and Male Genital Organs. WHO/IARC Classification oftumors, 4th Edition, Volume 8. IARC, Lyou, Reuter VE, pp 138-62.

Kang DE, Fitzsimons NJ, Presti JC, et al (2007). Risk stratification of men with Gleason score 7 - 10tumors by Primary and Secondary Gleason Score: Results from the SEARCH Database. Urology, 70, 277-82.

Matoso A, Epstein JI (2016). Grading of prostate cancer: Past, present, and future. Curr Urol Rep, 17, 576-8.

Melia J, Moseley R, Ball RY, et al (2006). A UK-based investigation of inter- and intra-observer reproducibility of Gleason grading of prostatic biopsies. Histopathology, 48, 644-54.

Mellinger GT, Gleason D, Bailar J (1967). The histology and prognosis of the prostate cancer. J Urol, 97, 331-7

Mellinger GT (1977). Prognosis of prostate carcinoma. Recent results. Cancer Res, 60, 61-72

Montironi R, Cheng L, Beltran AL, et al (2010) Original Gleason System Versus 2005 ISUP Modified Gleason System: The Importance of Indicating Which System Is Used in the Patient's Pathology and Clinical Reports. Eur Urol, 58, 369-73.

Montironi R, Mazzucchelli R, Beltran AL, Scarpelli M, Cheng L (2011). The Gleason grading system: where are we now?. Diagn Histopathol, 17, 419-27.

Pierorazio PM, Walsh PC, Partin AW, Epstein JI (2013). Prognostic Gleason grade grouping: data based on the modified Gleason scoring system. BJU Int, 111, 753-60.

Zabera P, Zhang J, Yilmaz A, Trpkor K (2009). The impact of the 2005 International Society of Urological Pathology (ISUP) consensus on Gleason grading in contemporary practice. Histopathology, 55, 384-91.

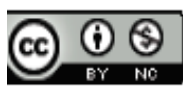

This work is licensed under a Creative Commons AttributionNon Commercial 4.0 International License. 